\title{
Primary Antiphospholipid Syndrome Associated with Diffuse Alveolar Hemorrhage and Pulmonary Thromboembolism
}

\author{
Takuma Isshiki, Keishi Sugino, Kyoko Gocho, Kenta Furuya, Hiroshige Shimizu, \\ Muneyuki Sekiya, Takanori Ohata, Tomohiro Wada, Kazutoshi Isobe, Susumu Sakamoto, \\ Yujiro Takai and Sakae Homma
}

\begin{abstract}
Antiphospholipid syndrome (APS) is clinically characterized by arterial or venous thrombosis; however, non-thromboembolic lung manifestations, such as diffuse alveolar hemorrhage (DAH), have also been previously reported. DAH is relatively common in APS patients with systemic lupus erythematosus, although it is rare in primary APS. We encountered a 78-year-old man who presented with hemoptysis and dyspnea. Chest CT showed diffuse ground-glass opacity with pulmonary thromboembolism. He was successfully treated with corticosteroids and heparin; however, DAH recurred after the corticosteroid treatment was stopped. The treatment was intricate due to the concurrent bleeding and thrombotic manifestations.
\end{abstract}

Key words: primary antiphospholipid syndrome, diffuse alveolar hemorrhage, pulmonary thromboembolism

(Intern Med 54: 2029-2033, 2015)

(DOI: 10.2169/internalmedicine.54.4058)

\section{Introduction}

Antiphospholipid syndrome (APS) is characterized by the clinical evidence of arterial or venous thrombosis, recurrent fetal loss, and thrombocytopenia accompanied by the elevation of at least one of the antiphospholipid antibodies or lupus anticoagulant testing $(1,2)$. APS is either primary or secondary to an underlying condition, most commonly systemic lupus erythematosus (SLE) (3). The spectrum of clinical manifestations seen in APS is wide and diverse. Most pulmonary manifestations result from the hypercoagulable state caused by antiphospholipid antibody (4), which can result in pulmonary thromboembolism, pulmonary hypertension, and acute respiratory distress syndrome-like disease. However, some manifestations, such as thrombocytopenia, cardiac valve disease, and diffuse alveolar hemorrhage (DAH) (5), have been reported even in the absence of thrombosis. DAH is associated with a number of clinical entities and several histologic subtypes. The most common un- derlying histologic subtype is connective tissue disorders, such as seropositive systemic vasculitis or SLE (6). DAH is relatively common in APS secondary to SLE (7); however, it is a rare manifestation in primary APS.

We herein report a case of DAH and pulmonary thromboembolism that developed concurrently in a patient with primary APS and was successfully treated with a combination of corticosteroids and heparin.

\section{Case Report}

A 78-year-old man with a 30-year history of hypertension was admitted to our hospital complaining of hemoptysis, a slight fever, and dyspnea for 10 days. He had a 28 pack/ year smoking history between the ages of 20 and 74 .

On admission, the patient's temperature was $37.6^{\circ} \mathrm{C}$ and his oxygen saturation was $92 \%$ in room air. Chest auscultation revealed fine crackles in the left lung fields, but no skin rash, skin ulcers, or joint swelling were present. The laboratory data on admission revealed a white blood cell count of 
Table. Laboratory Data on Admission.

\begin{tabular}{|c|c|c|c|c|c|}
\hline Blood chemistry & & Complete blood count & & Arterial blood gas & \\
\hline Total protein & $7.8 \mathrm{~g} / \mathrm{dL}$ & White blood cell count & $9,500 / \mu \mathrm{L}$ & $\mathrm{pH}$ & 7.409 \\
\hline Albumin & $3.2 \mathrm{~g} / \mathrm{dL}$ & Neutrophil & $81.3 \%$ & $\mathrm{PaCO}_{2}$ & $36.5 \mathrm{~mm} \mathrm{Hg}$ \\
\hline Aspartate aminotransferase & $27 \mathrm{U} / \mathrm{L}$ & Lymphocyte & $9.5 \%$ & $\mathrm{PaO}_{2}$ & $64.6 \mathrm{~mm} \mathrm{Hg}$ \\
\hline Alanine aminotransferase & $26 \mathrm{U} / \mathrm{L}$ & Eosinophil & $1.6 \%$ & & \\
\hline Alkaline phosphatase & $813 \mathrm{U} / \mathrm{L}$ & Monocyte & $7.4 \%$ & Immunology & \\
\hline Lactate dehydrogenase & $303 \mathrm{U} / \mathrm{L}$ & Red blood cell count & $410 \times 10^{4} / \mu \mathrm{L}$ & $\operatorname{IgG}$ & $1,391 \mathrm{mg} / \mathrm{dL}$ \\
\hline Urea nitrogen & $13 \mathrm{mg} / \mathrm{dL}$ & Hemoglobin & $13.2 \mathrm{~g} / \mathrm{dL}$ & $\mathrm{C} 3$ & $140 \mathrm{mg} / \mathrm{dL}$ \\
\hline Creatinine & $1.15 \mathrm{mg} / \mathrm{dL}$ & Hematocrit & $37.9 \%$ & $\mathrm{C} 4$ & $33 \mathrm{mg} / \mathrm{dL}$ \\
\hline Sodium & $133 \mathrm{mmol} / \mathrm{L}$ & Platelet count & $23.8 \times 10^{4} / \mu \mathrm{L}$ & CH 50 & $56.7 \mathrm{U} / \mathrm{mL}$ \\
\hline Potassium & $4.1 \mathrm{mmol} / \mathrm{L}$ & & & Antinuclear antibody & negative \\
\hline Chlorine & $101 \mathrm{mmol} / \mathrm{L}$ & Coagulation system & & Double standard DNA antibody & negative \\
\hline Calcium & $9.0 \mathrm{mg} / \mathrm{dL}$ & PT INR & 1.20 & PR3-ANCA & negative \\
\hline Phosphorus & $4.1 \mathrm{mg} / \mathrm{dL}$ & APTT & $23.7 \mathrm{sec}$ & MPO-ANCA & negative \\
\hline C-reactive protein & $11.1 \mathrm{mg} / \mathrm{dL}$ & Fibrinogen & $781 \mathrm{mg} / \mathrm{dL}$ & Lupus anticoagulant (PLCT) & $12.1 \mathrm{~s}$ \\
\hline Krebs von den Lungen- 6 & $269 \mathrm{U} / \mathrm{mL}$ & D-dimer & $51.8 \mu \mathrm{g} / \mathrm{mL}$ & Lupus anticoagulant (dRVVT) & negative \\
\hline Brain natriuretic peptide & $30.4 \mathrm{pg} / \mathrm{mL}$ & Fibrin degradation product & $6.4 \mu \mathrm{g} / \mathrm{mL}$ & A-CL $\beta 2$ GPI & negative \\
\hline Procalcitonin & $0.089 \mathrm{ng} / \mathrm{mL}$ & & & A-CL IgG antibody & negative \\
\hline
\end{tabular}

9,500 cells/ $\mu \mathrm{L}$, a C-reactive protein (CRP) level of $11.1 \mathrm{mg} /$ $\mathrm{dL}$, and a lactate dehydrogenase (LDH) level of $303 \mathrm{U} / \mathrm{L}$. Anemia and thrombocytopenia were not observed. The levels of Krebs von den Lungen-6 (KL-6) and procalcitonin were normal. Antinuclear antibody titers were negative, as were double-stranded DNA, antineutrophil cytoplasmic antibody (ANCA), and other autoantibodies. The prothrombin time (PT) and activated partial thromboplastin time (APTT) were normal. The result of a lupus anticoagulant test using hexagonal phospholipid neutralization was positive, although the results of tests for antiphospholipid antibodies were negative (Table). A chest radiograph showed the presence of diffuse infiltrate in the left lung field (Fig. 1A). CT and high-resolution CT (HRCT) revealed diffuse ground-glass opacity (GGO) in the left lung (Fig. 1B-D). Contrast CT showed pulmonary thrombosis in the right lower pulmonary artery (Fig. 1E) and deep vein thrombosis in the right popliteal vein. The bronchoalveolar lavage fluid (BALF) was bloody with hemosiderin-laden macrophages and no infectious organisms were identified; thus, we diagnosed the patient with DAH with pulmonary thromboembolism. The neutrophils (18\%), lymphocytes (26\%) and eosinophils (23\%) all showed increased levels in the BALF.

The patient was given methylprednisolone $(1,000 \mathrm{mg}$ daily) for 3 days, followed by prednisolone ( $25 \mathrm{mg}$ daily). Heparin therapy was started carefully in combination with corticosteroids for the pulmonary thromboembolism and deep vein thrombosis. The patient's hemoptysis resolved and oxygenation improved within several days of beginning the corticosteroid therapy. Chest CT showed improvement in GGO, and an ultrasonogram of the lower extremity showed improvement in deep vein thrombosis. The dose of corticosteroid was tapered, and heparin was switched to oral rivaroxaban. The patient was discharged with prescriptions for oral prednisolone $(10 \mathrm{mg})$ and rivaroxaban.

During the follow-up observation, prednisolone was ta- pered and then discontinued. The patient was on prednisolone therapy for a total of 3 months. One month after finishing the corticosteroid therapy, he developed a recurrence of dyspnea and hemoptysis. Chest CT showed bilateral GGO without pulmonary thromboembolism (Fig. 2). Bronchoscopy revealed bloody returns throughout the lavage with no evidence of infection. The result of a lupus anticoagulant test was again positive. The patient was diagnosed with recurrent DAH due to primary APS, and rivaroxaban was briefly discontinued due to the risk of increased hemorrhage. $\mathrm{He}$ was placed on high-dose intravenous methylprednisolone for 3 days, followed by daily prednisolone $(50 \mathrm{mg})$. Later, his oxygenation improved, and CT showed improvement in GGO. One week after resuming prednisolone, the patient developed sudden dysarthria. Brain diffusion-weighted magnetic resonance imaging (MRI) revealed multiple highintensity areas in the cerebellum and left temporal region (Fig. 3). He was diagnosed with an acute cerebral infarction and aspirin was started. The prednisolone dose was maintained at $10 \mathrm{mg}$ daily in combination with aspirin and rivaroxaban. No relapse of DAH or other thrombosis was observed during the 5 months of subsequent treatment.

\section{Discussion}

In this case of primary APS associated with DAH and pulmonary thromboembolism, bleeding and thrombotic manifestations developed concurrently. However, the patient was successfully treated with corticosteroids in combination with heparin. The DAH responded well to corticosteroid therapy without any immunosuppressive agents, although it recurred after therapy was stopped. Multiple thrombotic events developed during the clinical course.

APS is diagnosed based on the clinical evidence of arterial or venous thrombosis accompanied by an elevation of at least one of the antiphospholipid antibodies on direct anti- 


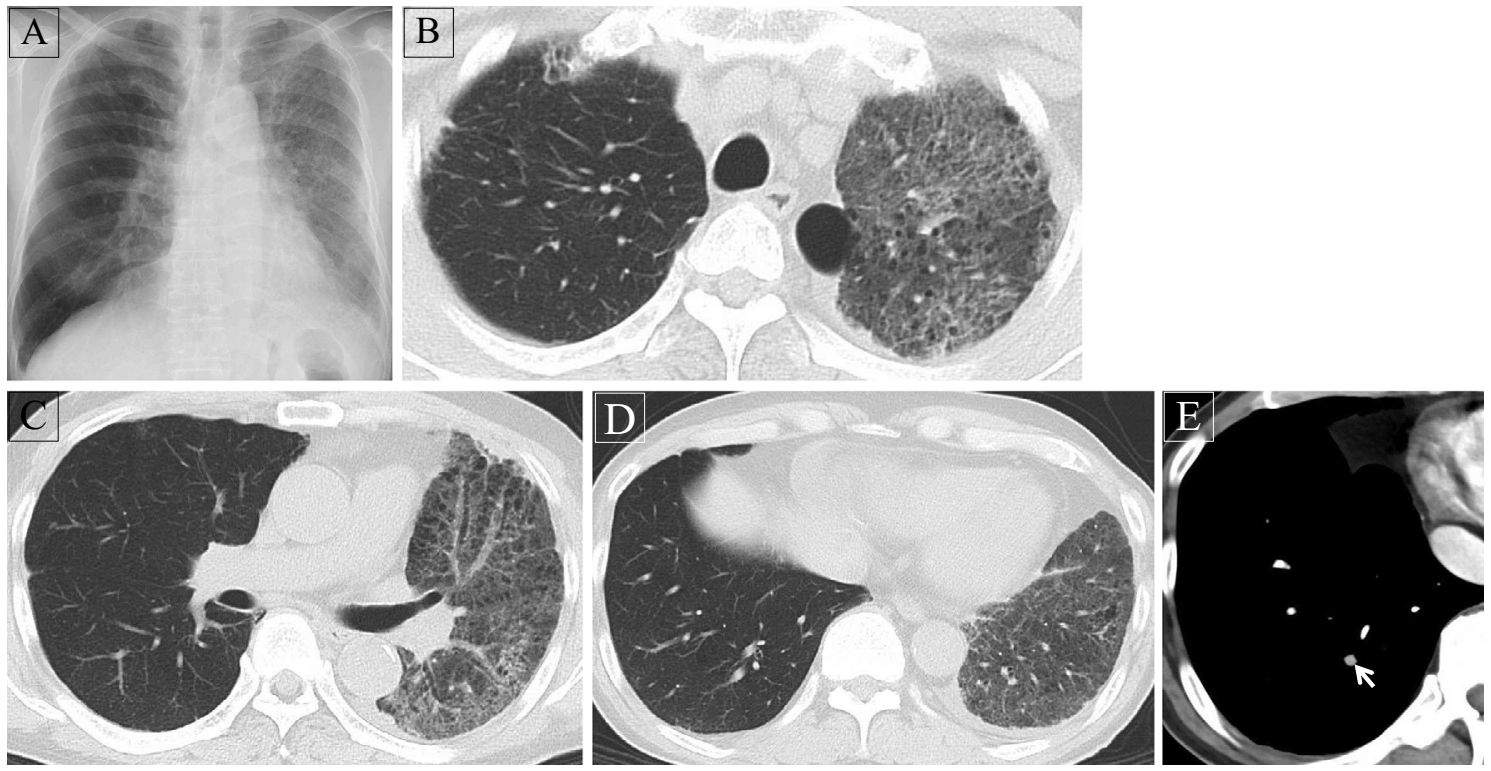

Figure 1. A chest radiograph on admission indicates diffuse infiltrates in the left lung field (A). Chest CT on admission indicates ground glass opacity distributed throughout the left lung field (B-D). Contrast chest CT indicates a pulmonary thromboembolism at the right lower branch of the pulmonary artery (arrow in E).
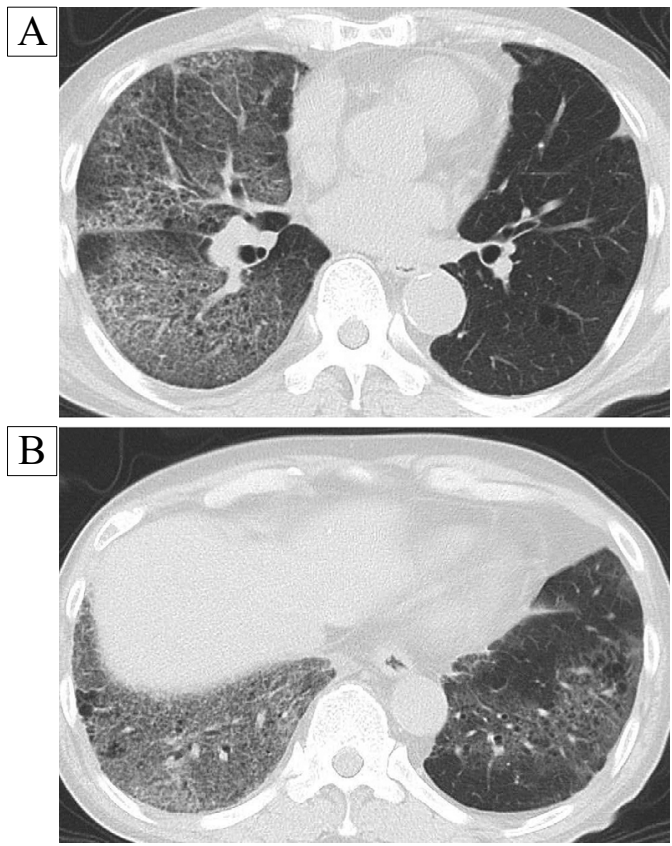

Figure 2. Chest CT indicates diffuse bilateral ground glass opacity without pulmonary thromboembolism (A, B).

body testing or lupus anticoagulant testing $(1,2)$. According to an international consensus statement, lupus anticoagulant positivity is confirmed when lupus anticoagulant is present in the plasma on two or more tests separated by an interval of at least 12 weeks (8). In the present case, lupus anticoagulant was positive only on a hexagonal phospholipid neutralization test; other antiphospholipid antibodies were negative. Past reports indicate that no single test has $100 \%$ sensitivity in detecting lupus anticoagulant (9). Additionally, Hughes and Khamashta described patients with clinical
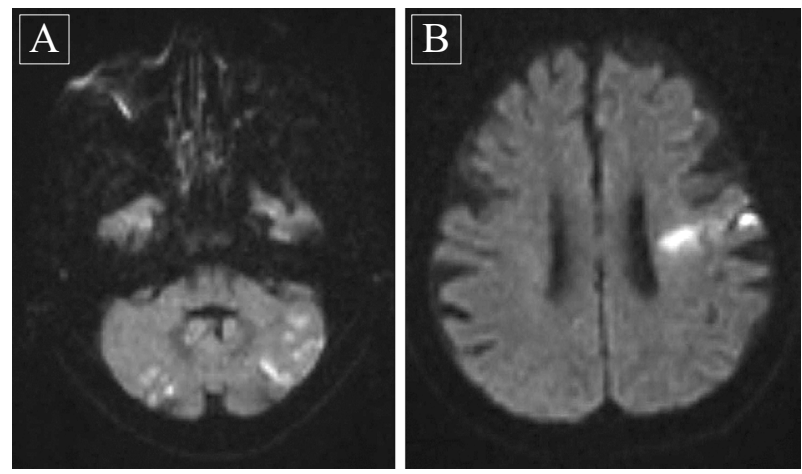

Figure 3. A diffusion-weighted magnetic resonance image of the brain indicates multiple high-intensity lesions in the cerebellum and left frontal lobe (A, B).

manifestations highly suggestive of antiphospholipid syndrome but with persistently negative results for lupus anticoagulant and antiphospholipid antibodies, a condition now referred to as "seronegative antiphospholipid syndrome" (10). Although the results of tests for other anticardiolipin antibodies and a lupus anticoagulant test were negative, our patient twice had a positive result for lupus anticoagulant on the hexagonal phospholipid neutralization tests given more than 12 weeks apart. Additionally, he developed multiple arterial and venous thromboses, which suggests a diagnosis of APS. We detected no specific clinical symptoms or serologic findings suggestive of other autoimmune diseases, such as SLE and ANCA-associated vasculitis. The diagnosis was therefore primary APS.

Because DAH is a rare manifestation in primary APS, the available literature is limited to case reports and small case series $(4,11-16)$. Although the precise pathogenic mecha- 
nisms are not well understood, it is believed that pulmonary capillaritis plays a role in the DAH pathogenesis. Dane and West reported four patients with primary APS who developed DAH, three of whom underwent open lung biopsy. The biopsy specimens showed neutrophilic infiltration of the alveolar septae consistent with pulmonary capillaritis and associated with hemorrhage into alveolar spaces. There was no evidence of vascular thrombosis, and the researchers hypothesized that DAH development was not due to thrombosis but rather to the antiphospholipid antibody-induced upregulation of endothelial cell adhesion molecules, with subsequent neutrophil recruitment and migration into the alveolar septae (4). Neutrophilia of BALF in our case was consistent with their hypothesis, however, lymphocytosis and eosinophilia were also observed. There is a possibility that an immune-mediated response was associated in our case.

Respiratory failure in our patient improved following high-dose corticosteroid therapy. Previous reports indicated that treatment of DAH in APS should start with corticosteroids for patients with severe respiratory distress (17). Other immunosuppressive agents, such as cyclophosphamide, azathioprine, and mycophenolate mofetil, have been used in an attempt to achieve complete remission. Additionally, some case reports noted that intravenous immunoglobulin treatment $(4,12,16)$, plasmapheresis $(13)$, and rituximab $(15,16)$ may be effective for patients who do not respond to corticosteroids. Cartin-Ceba et al. reported that immunosuppression with either cyclophosphamide or rituximab may have a higher likelihood of success (16). In the present case, DAH responded well to corticosteroids and is currently being controlled without other immunosuppressive agents. However, corticosteroid therapy alone may not be sufficient for the long-term control of DAH. Therefore, if it is not possible to control the disease or if other complications arise due to corticosteroid therapy, we intend to use immunosuppressive agents such as cyclophosphamide or rituximab.

In our patient, the treatment became intricate due to concurrent DAH and pulmonary thromboembolism. The ground glass opacity was distributed in almost the entire left lung field and pulmonary thromboembolism was limited to the right lower branch of the pulmonary artery. We considered that the main cause of respiratory failure in the patient was $\mathrm{DAH}$, however, there was deep vein thrombosis in the right popliteal vein and it may have led to the development of life-threatening pulmonary thromboembolism. Therefore, the administration of heparin was carefully initiated for the treatment of venous thrombosis and for the prevention of another thrombosis. Girardi et al. found that heparin decreased antiphospholipid antibody and may help moderate the disease (18). Although it is unclear whether heparin treatment results in the improvement of DAH caused by capillaritis, heparin may play a positive role, not only in treating thrombosis, but also in DAH.

Patients with APS develop arterial and venous thromboses. Previous reports indicate that the addition of an antiplatelet agent is a possible treatment for patients with recur- rent thrombosis during warfarin treatment (19). In the present case, rivaroxaban was the first drug used for venous thrombosis based on the EINSTEIN Investigators reports $(20,21)$. However, the patient later developed multiple cerebral infarctions. We used aspirin in combination with rivaroxaban to prevent subsequent arterial and venous thromboses. Under this regimen, thrombosis has been well controlled in our patient, without any adverse bleeding events.

The authors state that they have no Conflict of Interest (COI).

\section{References}

1. Asherson RA, Cervera R. Antiphospholipid syndrome. In: Textbook of Rheumatology. 5th ed. Kelley WN, Harris ED, Ruddy S, Sledge CB, Eds. Saunders, Philadelphia, 1997: 1057-1064.

2. Wilson WA, Gharavi AE, Koike T, et al. International consensus statement on preliminary classification criteria for definite antiphospholipid syndrome: report of an international workshop. Arthritis Rheum 42: 1309-1311, 1999.

3. Vianna JL, Khamashta MA, Orde-Ros J, et al. Comparison of the primary and secondary antiphospholipid syndrome: a European multicenter study of 114 patients. Am J Med 96: 3-9, 1994.

4. Deane KD, West SG. Antiphospholipid antibodies as a cause of pulmonary capillaritis and diffuse alveolar hemorrhage: a case series and literature review. Semin Arthritis Rheum 35: 154-165, 2005.

5. Erkan D, Lockshin MD. Non-criteria manifestations of antiphospholipid syndrome. Lupus 19: 424-427, 2010.

6. Lara AR, Schwarz MI. Diffuse alveolar hemorrhage. Chest 137: 1164-1171, 2010.

7. Zamora MR, Warner ML, Tuder R, Schwarz MI. Diffuse alveolar hemorrhage and systemic lupus erythematosus. Clinical presentation, histology, survival, and outcome. Medicine (Baltimore) 76: 192-202, 2007.

8. Miyakis S, LockShin MD, Atsumi T, et al. International consensus statement on an update of the classification criteria for definite antiphospholipid syndrome (APS). J Thromb Haemsot 4: 295-306, 2006.

9. Pengo V, Tripodi A, Reber G, et al. Update of the guidelines for lupus anticoagulant detection. Subcommittee on Lupus Anticoagulant/Antiphospholipid Antibody of the Scientific and Standardisation Committee of the International Society on Thrombosis and Haemostasis. J Thromb Haemost 7: 1737-1740, 2009.

10. Hughes GR, Khamashta MA. Seronegative antiphospholipid syndrome. Ann Rheum Dis 62: 1127, 2003.

11. Crausman RS, Achenbach GA, Pluss WT, O'brien RF, Jennings CA. Pulmonary capillaritis and alveolar hemorrhage associated with the antiphospholipid antibody syndrome. J Rheumatol 22: 554-556, 1995.

12. Gertner E. Diffuse alveolar hemorrhage in the antiphospholipid syndrome: spectrum of the disease and treatment. J Rheumatol $\mathbf{2 6}$ : 805-807, 1999.

13. Waterer GW, Latham B, Waring JA, Gabbay E. Pulmonary capillaritis associated with the antiphospholipid antibody syndrome and rapid response to plasmaapheresis. Respiorlogy 4: 405-408, 1999.

14. Suzuki A, Asazuma N, Kikuchi E, et al. "Possible primary antiphospholipid syndrome" with concurrent diffuse alveolar hemorrhaging and libman-sacks endocarditis mimicking catastrophic antiphospholipid syndrome. Intern Med 51: 813-816, 2012.

15. Scheiman Elazary A, Klahr PP, Hershko AY, Dranitzki Z, Rubinow A, Naparstek Y. Rituximab induces resolution of recurrent diffuse alveolar hemorrhage in a patient with primary antiphospholipid antibody syndrome. Lupus 41: 438-440, 2012. 
16. Cartin-Ceba R, Peikert $T$, Ashrani A, et al. Primary antiphospholipid syndrome-associated diffuse alveolar hemorrhage. Arthritis Care Res (Hoboken) 66: 301-310, 2014.

17. Espinosa G, Cervera R, Font J, Asherson RA. The lung in the antiphospholipid syndrome. Ann Rheum Dis 61: 195-198, 2002.

18. Girardi G, Redecha P, Salmon JE. Heparin prevents antiphospholipid antibody-induced fetal loss by inhibiting complement activation. Nat Med 10: 1222-1226, 2004.
19. Lim W, Crowther MA, Eikelboom JW. Management of antiphospholipid antibody syndrome. A systematic review. JAMA 295: 1050-1057, 2006.

20. The EINSTEIN Invetigators. Oral rivaroxaban for symtopmatic venous thromboembolism. N Engl J Med 363: 2499-2510, 2010.

21. The EINSTEIN Investigators. Oral rivaroxaban for the treatment of symptomatic pulmonary embolism. N Engl J Med 366: $1287-$ 1297, 2012.

(C) 2015 The Japanese Society of Internal Medicine http://www.naika.or.jp/imonline/index.html 